\title{
Calculating Colimits Compositionally
}

\author{
Robert Rosebrugh $^{1}$, Nicoletta Sabadini ${ }^{2}$, and Robert F.C. Walters ${ }^{2, \star}$ \\ ${ }^{1}$ Department of Mathematics and Statistics \\ Mt. Allison University, Sackville, \\ New Brunswick, Canada \\ 2 Dipartimento di Scienze delle Cultura, Politiche e dell'Informazione, \\ Università dell'Insubria, Italy
}

\begin{abstract}
We show how finite limits and colimits can be calculated compositionally using the algebras of spans and cospans, and give as an application a proof of the Kleene Theorem on regular languages.
\end{abstract}

\section{Introduction}

In Computer Science:

The state spaces of systems are often described by finite limits or colimits in a category $\mathbf{E}$ parametrized by a graph $G$ which describes the underlying geometry of the system. It is desirable that there is also an algebraic description, so that the limit or colimit is described by an expression, rather than geometrically.

This goes back to the beginnings of computer science, where (i) a program may be described either by a flow chart (goto's), or program text (while) (BöhmJacopini), (ii) a language may be specified by an automaton or an expression (Kleene). And of course it is present in innumerable areas of computer science (Petri nets versus process algebras, wysiwig versus markup, graph versus term rewriting, etc.) and mathematics.

\section{In Category Theory:}

Finite limits and colimits are parametrized by graphs; that is, geometrically. We show that they can also be described by expressions in an algebra. As an application we prove Kleene's theorem.

Perhaps the first proposal for a strict relation between graphic and algebraic/categorical descriptions arose in the work of R. Penrose 20 in his graphical description of the tensor calculus in 1971. C.C. Elgot [6] began the algebraicization of flowcharts and circuits introducing a categorical algebra which contained three basic operations; in circuit terminology - series and parallel composition, and feedback. In subsequent work this algebra has been intensively developed by S.L. Bloom and Z. Esik, with the state of progress being recorded in their monograph [2].

\footnotetext{
* The authors gratefully acknowledge financial support from the Universitá dell'Insubria, the Italian Government PRIN project ART (Analisi di sistemi di Riduzione mediante sistemi di Transizione), and the Canadian NSERC.
} 
In a parallel development G.M. Kelly and M.L. Laplaza [16 gave a graphical account of compact closed monoidal categories. Following [25], A. Joyal and R.H. Street 9 discovered the notion of braided monoidal category, a major impulse towards the study of geometry and higher dimensional categories. Among the many categorical developments was the discovery in [4. of the Frobenius equation and the recognition by Joyal of its importance in 2-dimensional cobordism theory [17.

Ugo Montanari and his collaborators have played a fundamental role in related developments in computer science, beginning with the fundamental paper [19] relating Petri nets and monoidal categories. Gadducci and Heckel [7, 8] discovered that a category of cospans of graphs is a free symmetric monoidal category with appropriate structure and axioms (one being the Frobenius axiom (see also [22]), and gave an algebraic formulation of double-pushout graph rewriting.

In the last ten years there has been a flowering of applications of monoidal categories in geometry, physics and computer science. We cite just three directions in computer science [10, [3, 1].

The algebra in which finite limits and colimits in $\mathbf{E}$ may be expressed compositionally is an appropriate structure on spans and cospans in the category. This fact is a partial explanation for the algebra of spans and cospans introduced in [12, [14] and developed in various papers, such as [13], [15, 21].

This note is an expanded version of a lecture 23. to Category Theory 2007, Carvoeiro, Portugal, 18th June 2007. A more detailed version with full proofs is in preparation [24.

\section{What Algebra?}

Assume now $\mathbf{E}$ is a category with finite colimits. What is the algebra in which finite colimits in $\mathbf{E}$ can be described by expressions?

It is cospan $(\mathbf{E})$, considered as a symmetric monoidal category in which each object has a commutative separable algebra structure. We call a category with such a structure wscc (well-supported compact closed [26]). To be precise:

Definition 1. A commutative separable algebra 4 4 in a symmetric monoidal category is an object $A$ equipped with four arrows

$$
!: I \longrightarrow A, \nabla: A \otimes A \longrightarrow A, \mathrm{i}: A \longrightarrow I, \Delta: A \longrightarrow A \otimes A
$$

such that $(A, \nabla, !)$ forms a commutative monoid, $(A, \triangle, \mathrm{i})$ forms a cocommutative comonoid, and the following three axioms hold

$$
\begin{gathered}
\left(1_{A} \otimes \nabla\right)\left(\Delta \otimes 1_{A}\right)=\Delta \nabla=\left(\nabla \otimes 1_{A}\right)\left(1_{A} \otimes \Delta\right), \\
\nabla \Delta=1_{A} .
\end{gathered}
$$

We can draw a picture of the last three extra axioms, namely: 

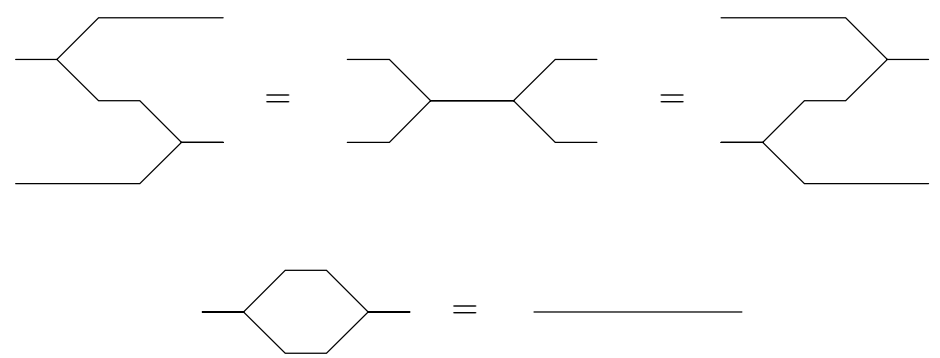

The wscc structure induces a self-dual compact closed structure on the category, and we denote the units and counits of this structure as

$$
\eta_{A}: I \rightarrow A \otimes A(=\Delta \cdot !), \varepsilon_{A}: A \otimes A \rightarrow I(=i \cdot \nabla) .
$$

For some background to these axioms see also [17.

\subsection{The wscc Structure on Span and Cospan Categories}

We will describe the wscc structure on $\operatorname{cospan}(\mathbf{E})$ for $\mathbf{E}$ a finitely cocomplete category - the dual structure on $\operatorname{span}(\mathbf{E})$ will then be clear.

An object of $\operatorname{cospan}(\mathbf{E})$ is an object of $\mathbf{E}$; an arrow of $\operatorname{cospan}(\mathbf{E})$ from $A$ to $B$ is an isomorphism class of cospans from $A$ to $B$; that is, of pairs of arrows

$$
\alpha_{1}, \alpha_{2}: A \rightarrow R \leftarrow B
$$

We will use the notation $\alpha_{1}, \alpha_{2} ; A \longleftrightarrow B$ to distinguish cospans from arrows in E. However given any arrow $f: A \rightarrow B$ there are special cospans denoted $\boldsymbol{f}=f, 1_{B}: A \longleftrightarrow B$ and $f^{o}=1_{B}, f: B \longleftrightarrow A$. Composition of cospans is by pushout. Now to describe the wscc structure of $\operatorname{cospan}(\mathbf{E})$. The monoidal structure is sum. The special arrows

$$
!: I \longrightarrow A, \nabla: A \otimes A \longrightarrow A, \mathrm{i}: A \longrightarrow I, \Delta: A \longrightarrow A \otimes A
$$

are (using $\nabla$ both for the codiagonal in $\mathbf{E}$ and the structure in $\operatorname{cospan}(\mathbf{E})$, and similarly overloading the symbol !)

$!=!: 0 \longleftrightarrow A, \nabla=\nabla: A+A \longleftrightarrow A, \mathrm{i}=!^{\circ}: A \longleftrightarrow 0, \Delta=\nabla^{o}: A \longleftrightarrow A+A$

\section{$2.2 \operatorname{Cspn}(\operatorname{Graph} /|\mathrm{E}|)$}

Let Graph be the category of finite graphs, let $|\mathbf{E}|$ be the underlying graph (possibly infinite) of $\mathbf{E}$. Consider Graph $/|\mathbf{E}|$, the category with objects diagrams in $\mathbf{E}$, and morphisms compatible graph morphisms. Then $C \operatorname{spn}(G r a p h /|\mathbf{E}|)$ is the full subcategory of cospan $($ Graph $/|\mathbf{E}|)$ whose objects are discrete diagrams in $\mathbf{E}$.

Notice that colimits in this category are calculated as in Graph, and are unrelated to colimits in $\mathbf{E}$. 
We will denote diagrams using set-theoretical notation; for example

$$
\{A \underset{g}{\stackrel{f}{\longrightarrow}} B\}
$$

denotes the diagram with two parallel arrows.

It will be useful to introduce a way of picturing arrows in $C \operatorname{spn}(\operatorname{Graph} /|\mathbf{E}|)$ (engineering notation). Represent the objects in the centre graph of the cospan as points, and arrows in the centre as components with one input (to the left) and one output (to the right) joined to those points which are the domain and codomain of the arrow. Represent the graph morphisms of the cospan as input and output wires of the whole picture.

Example 1. Consider the following cospan of diagrams

$$
\{A\} \longrightarrow\{A \underset{g}{\stackrel{h}{\rightleftarrows}} B \stackrel{h}{\longrightarrow} C\} \longleftarrow\{C\} .
$$

This cospan could be pictured as

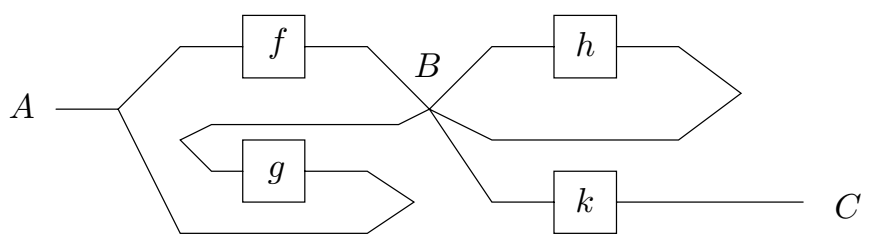

\section{The Theorem}

Taking colimit of diagrams in $\mathbf{E}$ induces a functor

$$
\text { colim }: G r a p h /|\mathbf{E}| \rightarrow \mathbf{E} .
$$

Theorem 1. The functor colim $: G r a p h /|\mathbf{E}| \rightarrow \mathbf{E}$ extends to a functor

$$
\operatorname{colim}: C \operatorname{spn}(\operatorname{Graph} /|\mathbf{E}|) \rightarrow \operatorname{cospan}(\mathbf{E})
$$

which preserves the wscc structure.

The definition of the extended colimit is just applying colimit to cospans. It is straightforward that this colim preserves the constants of wscc structure of $\operatorname{Cspn}(\operatorname{Graph} /|\mathbf{E}|)$, and that colim of the cospan of the diagram

$$
\{A\} \longrightarrow\{A \stackrel{f}{\longrightarrow} B\} \longleftarrow\{B\}
$$

is $\boldsymbol{f}: A \longleftrightarrow B$. The fact that colim preserves the tensor is also clear. What remains to prove is the fact that colim is a functor - we outline the proof below. 
Another special case of colim is worth remarking. Consider a cospan in which the centre diagram is also discrete, so that we may consider the cospan to be of the form

$$
\left\{A_{i}\right\}_{(i \in I)} \stackrel{\phi: I \rightarrow J}{\longrightarrow}\left\{B_{j}\right\}_{(j \in J)} \stackrel{\psi: J \leftarrow K}{\longleftarrow}\left\{C_{k}\right\}_{(k \in K)} .
$$

Then colimit applied to this cospan is

$$
\Sigma_{i \in I} A_{i} \stackrel{\operatorname{colim}(\phi)}{\longrightarrow} \Sigma_{j \in J} B_{j} \stackrel{\operatorname{colim}(\psi)}{\longleftarrow} \Sigma_{k \in K} C_{k},
$$

where $\operatorname{colim}(\phi) \cdot i n j_{i}=i n j_{\phi(i)}(i \in I)$ and $\operatorname{colim}(\psi) \cdot i n j_{k}=i n j_{\psi(k)}(k \in K)$

Remark 1. $\operatorname{Cspn}(\operatorname{Graph} /|\mathbf{E}|)$ is the result of freely adding wscc category structure to the graph $|\mathbf{E}|$ (a special case of this result was proved in [22]). This means that diagrams in $|\mathbf{E}|$ may be written as expressions in the wscc structure of $\operatorname{Cspn}(\operatorname{Graph} /|\mathbf{E}|)$ with constants being the cospans of the form $\boldsymbol{f}$ for arrows $f$ of $|\mathbf{E}|$.

Then colim preserves wscc expressions, so the colimit of any diagram may be written as an expression in cospan $(\mathbf{E})$. This is the compositionality of the calculation of colimits, mentioned in the title.

\subsection{The Example of Coequalizers}

Consider the following cospan of diagrams in $\mathbf{E}$ :

$$
\{A\} \longrightarrow\{A \underset{g}{\stackrel{f}{\longrightarrow}} B\} \longleftarrow\{B\} .
$$

The cospan of diagrams may be pictured, as described above, as

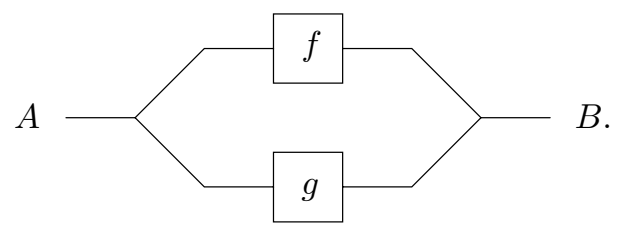

It is clear from the picture that the cospan may be expressed as the following composite in $\operatorname{Cspn}(\operatorname{Graph} /|\mathbf{E}|)$ :

$$
\{A\} \stackrel{\Delta}{\longleftrightarrow}\{A\}+\{A\} \stackrel{\{f\}+\{g\}}{\longleftrightarrow}\{B\}+\{B\} \stackrel{\nabla}{\longleftrightarrow}\{B\} .
$$

Applying colimit we see that the coequalizer of $f$ and $g$ may be expressed as the composite in cospan $(\mathbf{E})$

$$
A \stackrel{\Delta}{\longleftrightarrow} A+A \stackrel{f+\boldsymbol{g}}{\longrightarrow} B+B \stackrel{\nabla}{\longrightarrow} B .
$$


The composite of these three cospans is the pushout $\mathrm{Q}$ of the following diagram in $E$

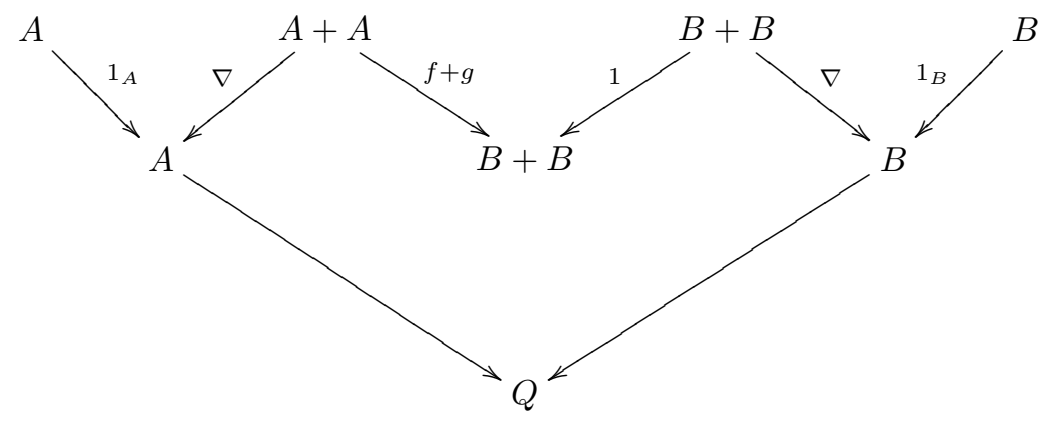

It is easy to verify directly that $Q$ so defined is the coequalizer of $f$ and $g$.

Example 2. By the same kind of reasoning the colimit of the diagram in example 1 may be given by the expression of cospans in $\mathbf{E}$

$\left(\epsilon_{B}+C\right) \cdot(\boldsymbol{h}+B+\boldsymbol{k}) \cdot(\Delta+B) \cdot \Delta \cdot\left(\nabla+\epsilon_{A}\right) \cdot(\boldsymbol{f}+B+\boldsymbol{g}+A) \cdot\left(A+\eta_{B}+A\right) \cdot \Delta$.

Remark 2. Theorem 1 has an analogue for limits. If $\mathbf{E}$ has finite limits the functor $l i m:(\operatorname{Graph} /|\mathbf{E}|)^{o p} \rightarrow \mathbf{E}$ extends to a functor

$$
\lim : C \operatorname{spn}(\operatorname{Graph} /|\mathbf{E}|) \rightarrow \operatorname{span}(\mathbf{E})
$$

which preserves the wscc structure. This permits the compositional calculation of finite limits in E. In fact the equalizer of two arrows $A \underset{g}{\stackrel{f}{\longrightarrow}} B$ may be calculated by the same expression as that of the coequalizer above but evaluated in $\operatorname{span}(\mathbf{E})$ rather than cospan $(\mathbf{E})$, since in both cases the expression is determined by the wscc structure of $C \operatorname{spn}(\operatorname{Graph} /|\mathbf{E}|)$.

\subsection{Sketch of Proof of Theorem}

The main point to check in showing that colim is a monoidal functor is (a special case of) the following:

Consider a diagram $D$ of diagrams in $\mathbf{E}$ parametrized by a graph $G$; that is, a graph morphism $D: G \rightarrow G r a p h /|\mathbf{E}|$. We can do two things.

(1) Calculate first the colimit of $D$ in $G r a p h /|\mathbf{E}|$ to obtain a diagram in $\mathbf{E}$ of which we may then take the colimit in $\mathbf{E}$, that is calculate

$$
\operatorname{colim}_{\mathbf{E}}\left(\operatorname{colim}_{G r a p h /|\mathbf{E}|}(D)\right) \text {. }
$$

(2) Calculate the colimit of

$$
G \stackrel{D}{\longrightarrow} G \mathrm{raph} / \mathbf{E} \stackrel{\text { colim }}{\longrightarrow} \mathbf{E}
$$

that is, calculate $\operatorname{colim}_{\mathbf{E}}\left(\operatorname{colim}_{\mathbf{E}} \cdot D\right)$. 


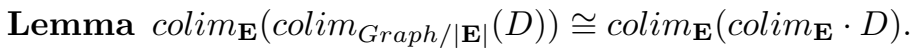

Sketch of proof.

It suffices to show for any $X \in \mathbf{E}$ a bijection between cocones

$$
\operatorname{colim}_{G r a p h /|\mathbf{E}|}(D) \longrightarrow X
$$

and cocones

$$
\operatorname{colim}_{\mathbf{E}} \cdot D \longrightarrow X
$$

But it is not hard to show that both of these are equivalent to a "compatible family" of cocones

$$
D(g) \longrightarrow X \quad(g \in G) .
$$

A Very Special Case. Consider a diagram $D$ of diagrams in $\mathbf{E}$, namely

$$
D=\{\{A\}\{B C\}\} .
$$

Then

$$
\operatorname{colim}_{\mathbf{E}}\left(\operatorname{colim}_{\text {Graph } / \mathbf{E} \mid}(D)\right)=\operatorname{colim}_{\mathbf{E}}(\{A B C\})=A+B+C,
$$

whereas

$$
\operatorname{colim}_{\mathbf{E}}\left(\operatorname{colim}_{\mathbf{E}} \cdot D\right)=\operatorname{colim}_{\mathbf{E}}(\{A B+C\})=A+(B+C) .
$$

The lemma says exactly that the triple sum may be formed by repeated double sums, which has as a consequence the associative law for sums. It is clear that the general form of the lemma implies many further "associative laws" - any two wscc expressions which yield the same diagram evaluate to the same object in $\operatorname{cospan}(\mathbf{E})$.

\subsection{Example of Theorem}

A general cospan in $C \operatorname{spn}(G r a p h /|\mathbf{E}|)$ from $\varnothing$ to $\varnothing$ with centre $D$ may be constructed by taking the disjoint sum of all the arrows, and then equating vertices appropriately. This yields a formula for the general colimit of a finite diagram as follows. Let $\Sigma_{\text {dom }}$ denote the graph $\sum_{\alpha \in D}\{\operatorname{dom}(\alpha)\}$ and $\Sigma_{\text {cod }}$ denote the graph $\sum_{\alpha \in D}\{\operatorname{codom}(\alpha)\}$. Let $\Sigma \alpha$ denote the graph $\sum_{\alpha \in D}\{\alpha\}$. Let $\Sigma_{o b j}$ denote the diagam consisting of all the objects in the $D$. Finally, let $i_{d o m}$ and $i_{\text {cod }}$ denote the discrete cospans corresponding to the domain and codomain functions on the arrows of the graph parametrizing $D$. Then the cospan may be written

$$
\{\} \longleftrightarrow \Sigma_{\text {dom }}+\Sigma_{\text {dom }} \stackrel{i_{\text {cod }} \cdot\left(\sum \alpha\right)+i_{\text {dom }}}{\longleftrightarrow} \Sigma_{o b j}+\Sigma_{o b j} \longleftrightarrow \epsilon \quad\{\} .
$$

Evaluating this formula instead in cospan $(\mathbf{E})$ gives the classical formula for colimits in terms of the coequalizer of two arrows from $\sum_{\alpha \in D} \operatorname{dom}(\alpha)$ to $\sum_{A \in D} A$ ( $\alpha$ arrow in $D, A$ object in $D$ ). 


\section{Limits and Colimits of Monoidal Diagrams}

Systems in computer science are not usually constructed from parts with one input and one output, like arrows in a graph. Components have multiple inputs and outputs; that is, they are arrows in a monoidal graph.

Definition 2. $A$ monoidal graph $\left(A, V, d_{0}, d_{1}\right)$ consists of a set $V$ of vertices, and a set $A$ of arcs and two functions $d_{0}, d_{1}: A \longrightarrow V^{*}\left(V^{*}\right.$ the free monoid on $V)$. $A$ morphism of monoidal graphs $\phi=\left(\phi_{0}, \phi_{1}\right)$ from $\left(A, V, d_{0}, d_{1}\right)$ to $\left(B, W, d_{0}, d_{1}\right)$ consists of two functions $\phi_{1}: A \rightarrow B$ and $\phi_{0}: V \rightarrow W$ such that $\phi_{0}^{*} d_{0}=d_{0} \phi_{1}, \phi_{0}^{*} d_{1}=d_{1} \phi_{1}$. We denote the category (actually a presheaf category) of monoidal graphs as MonGraph. There is an obvious notion then of a monoidal diagram in a monoidal category since any monoidal category has an underlying monoidal graph.

Definition 3. Let $\mathbf{E}$ be a category with finite colimits, regarded as a monoidal category with sum as tensor. A cocone $q$ of a monoidal diagram $D$ to an object $X$ is a family of arrows $\left(q_{i}: A_{i} \longrightarrow X\right) \quad\left(A_{i}\right.$ objects of the diagram $\left.D\right)$ such that for any arrow $f: A_{i_{1}}+A_{i_{2}}+\cdots+A_{i_{m}} \rightarrow A_{j_{1}}+A_{j_{1}}+\cdots+A_{j_{n}}$ in the diagram

$$
\left(q_{j_{1}}\left|q_{j_{2}}\right| q_{j_{n}}|\cdots| q_{j_{n}}\right) \cdot f=\left(q_{i_{1}}\left|q_{i_{2}}\right| q_{i_{3}} \cdots \mid q_{i_{m}}\right) .
$$

A colimit of monoidal diagram $D$ is an object $C$ with a cocone $q$ from $D$ which is univeral; that is, any cocone to an object $X$ factors uniquely through $q$.

\subsection{Cspn(MonGraph/|E $\mid)$}

Let $\mathbf{E}$ be a category with finite colimits, regarded as a monoidal category with sum as tensor, and let $|\mathbf{E}|$ denote the underlying monoidal graph of $\mathbf{E}$. Then $\operatorname{Cspn}($ MonGraph $/|\mathbf{E}|)$ denotes the full subcategory of $\operatorname{cospan}(\operatorname{MonGraph} /|\mathbf{E}|)$ whose objects are discrete diagrams in E. Just as with $C \operatorname{spn}(G r a p h /|\mathbf{E}|)$ we may picture arrows in $C \operatorname{spn}(M o n G r a p h /|\mathbf{E}|)$, the only difference being that components may have several input and output wires. Monoidal colimits may also be calculated compositionally, in the algebra cospan $(\mathbf{E})$, by a result analogous to Theorem 1. Taking the monoidal colimit of diagrams in $\mathbf{E}$ induces a functor

$$
\text { moncolim : MonGraph } /|\mathbf{E}| \rightarrow \mathbf{E} \text {. }
$$

Theorem 2. The functor moncolim : MonGraph $/|\mathbf{E}| \rightarrow \mathbf{E}$ extends to a functor

$$
\text { moncolim : Cspn (MonGraph } /|\mathbf{E}|) \rightarrow \operatorname{cospan}(\mathbf{E})
$$

which preserves the wscc structure.

We look at one example only. 


\subsection{Example}

Consider the following cospan of monoidal diagrams $D$ in $\mathbf{E}$ : the centre is the diagram with three objects $A, B, C$, and one arrow $f: A+C \rightarrow B+C$; the left hand side is the diagram $\{A\}$; the right hand side is the diagram $\{B\}$. Pictured, the cospan is

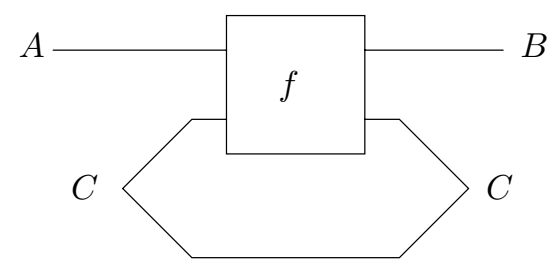

From the picture we see immediately that this cospan of diagrams is expressible as a composite in $C \operatorname{spn}($ MonGraph/|E $\mid)$, namely

$$
\{A\} \stackrel{\{A\}+\eta_{\{C\}}}{\longleftrightarrow}\{A\}+\{C\}+\{C\} \stackrel{\{f\}+\{C\}}{\longleftrightarrow}\{B\}+\{C\}+\{C\} \stackrel{\{B\}+\epsilon_{\{C\}}}{\longleftrightarrow}\{B\} .
$$

Applying monoidal colimit yields the fact that the monoidal colimit of the original diagram is the following composite in $\operatorname{cospan}(\mathbf{E})$ :

$$
A \stackrel{A+\eta_{C}}{\longrightarrow} A+C+C \stackrel{f+C}{\longrightarrow} B+C+C \stackrel{B+\epsilon_{C}}{\longrightarrow} B .
$$

Hence the colimit of the original diagram can be calculated as the pushout below.

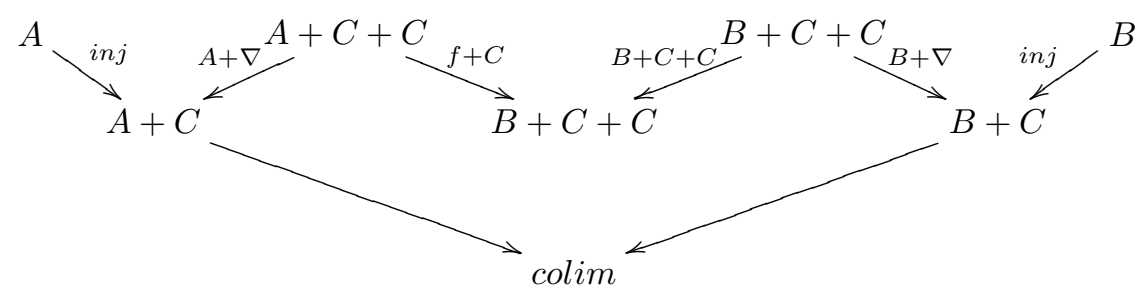

The colimit consists of orbits of $A+B+C$ under $f$. The pullback of the resulting cospan is the partial function obtained by iterating $f$.

\section{The Kleene Theorem}

Theorem 3. (Kleene)

The languages recognized by finite state automata are the closure of singletons under union, concatenation and iteration.

To prove this classical theorem the category $\mathbf{E}$ we consider is $\wp\left(\Sigma^{*}\right)$-Cat, categories enriched in $\Sigma$-languages. There is a composite of wscc functors

$$
C \operatorname{spn}(\operatorname{Graph} / \Sigma) \stackrel{\Phi_{1}}{\longrightarrow} \operatorname{Cspn}\left(\operatorname{Graph} / \wp\left(\Sigma^{*}\right)\right) \stackrel{\Phi_{2}}{\longrightarrow} \operatorname{cospan}\left(\wp\left(\Sigma^{*}\right)-C a t\right) .
$$


which takes a labelled graph (with input and output states) to the $\wp\left(\Sigma^{*}\right)$ category whose homs are the languages traced out from the domain to the codomain. The existence of wscc functor $\Phi_{1}$ is implied by [22], and of $\Phi_{2}(=$ colim) by Theorem 1 .

This is already a Kleene-type theorem since, conceptually, the Kleene Theorem says that behaviour is an operation-preserving morphism from an algebra of systems to an algebra of possible behaviours, which implies that the perceived behaviours are the smallest class of possible behaviours closed under operations. In this case, the algebra of systems, that is the left-hand side, is generated as a wscc category by single labelled edges, and hence the image on the right-hand side is also generated as a wscc category by singleton languages.

However it is not the classical Kleene theorem, since the right-hand side does not consist of single languages and the wscc operations of $\operatorname{cospan}\left(\wp\left(\Sigma^{*}\right)-C a t\right)$ are not the Kleene operations. Further the functor does not lose internal states.

To obtain a theorem closer to the classical Kleene theorem we consider corelations between $\wp\left(\Sigma^{*}\right)$ categories, by which we mean cospans which are jointly bijective on objects. Then we compose the above wscc functor $\Phi_{2} \Phi_{1}$ with a further wscc functor

$$
\Phi_{3}: \operatorname{cospan}\left(\wp\left(\Sigma^{*}\right)-C a t\right) \longrightarrow \operatorname{corel}\left(\wp\left(\Sigma^{*}\right)-C a t\right)
$$

which uses the bijective-on-objects fully-faithful factorization to obtain from a cospan of $\wp\left(\Sigma^{*}\right)$-categories a corelation of $\wp\left(\Sigma^{*}\right)$-categories.

The final composite

$$
\Phi_{3} \Phi_{2} \Phi_{1}: C \operatorname{spn}(\operatorname{Graph} / \Sigma) \longrightarrow \operatorname{corel}\left(\wp\left(\Sigma^{*}\right)-C a t\right)
$$

takes a labelled graph with initial and final states to the category with objects only the initial and final states, and whose homs are the languages traced out.

To finish a proof of the classical Kleene theorem we need to show that the wscc operations in $\operatorname{corel}\left(\wp\left(\Sigma^{*}\right)-C a t\right)$, at the level of languages (homs), may be expressed in terms of union, concatenation and ( )*.

Clearly the operation of tensor of corelations does not change the languages which occur as homs. The problem is the composition. But in a wscc category the composition of two arrows may be expressed in terms of the tensor and composition with the constants of the compact closed structure; pictured, this is the fact that:

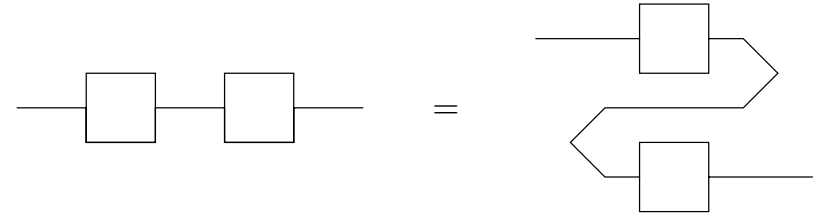

So the general operation of composition in $\operatorname{corel}\left(\wp\left(\Sigma^{*}\right)\right.$-Cat $)$ may be reduced to the very special case of the colimit identifying two objects in a category. 
Consider a $\wp\left(\Sigma^{*}\right)$-category $X$ containing two objects $x$ and $y$. The colimit category $X^{\prime}=X /(x=y)$ has hom $X^{\prime}(z, w)$ equal to

$$
X(z, w) \cup(X(z, x) \cup X(z, y)) \cdot(X(x, x) \cup X(x, y) \cup X(y, x) \cup X(y, y))^{*} \cdot(X(x, w) \cup X(y, w))
$$

expressible using only Kleene operations. Hence the result.

This proof is very close to one of the usual proofs of Kleene (if you strip the superstructure). Notice that the passage $\Phi_{1}$ permits the introduction of $\varepsilon$ moves; that is, homs which consist of the empty word. The superstructure has the advantage of suggesting needed generalizations, for example, to parallelism.

\section{Comments}

The theorem we have described concerns calculating colimits as objects, not as functors. We have not shown the compositionality of morphisms between colimits. We believe that this is connected with the algebra of span and cospan as symmetric monoidal bicategories, rather than as categories. We have made initial progress in understanding this question in [18, by considering a very special case, where we identify the role of 2-separable object.

There is a more precise relation between this work and the paper of Ugo Montanari and José Meseguer [19. Monoidal graphs may be thought of as Petri nets without markings. Processes of a net $G$ are arrows in the free symmetric monoidal category on $G$. But in 5 we show that the free symmetric monoidal category on $G$ is an easily identifiable subcategory of $C \operatorname{spn}(M o n G r a p h / G)$, with the same objects as $C \operatorname{spn}(M o n G r a p h / G)$, but with arrows being cospans of "linear monoidal diagrams", that is, those parametrized by monoidal graphs without loops, forking or merging. Linear monoidal diagrams are a generalization of paths in a graph. (Other classes of free categories are similarly obtainable by specializing the type of graphs in the cospans).

\section{References}

1. Abramsky, S., Coecke, B.: A Categorical Semantics of Quantum Protocols. In: Proceedings of the 19th Annual IEEE Symposium on Logic in Computer Science: LICS 2004, pp. 415-425. IEEE Computer Society, Los Alamitos (2004)

2. Bloom, S.L., Esik, Z.: Iteration Theories: the equational logic of iterative processes. EATCS Monographs in Theoretical Computer Science. Springer, Heidelberg (1993)

3. Bruni, R., Gadducci, F., Montanari, U.: Normal forms for algebras of connection. Theor. Comput. Sci. 286(2), 247-292 (2002)

4. Carboni, A., Walters, R.F.C.: Cartesian bicategories I. Journal of Pure and Applied Algebra 49, 11-32 (1987)

5. de Francesco Albasini, L., Rosebrugh, R., Sabadini, N., Walters, R.F.C.: Cospans and free symmetric monoidal categories (in preparation)

6. Elgot, C.C.: Monadic computation and iterative algebraic theories, Logoc Colloquium 1973, Studies in Logic 80, pp. 175-230. North Holland, Amsterdam (1975) 
7. Gadducci, F., Heckel, R.: An inductive view of graph transformation. In: ParisiPresicce, F. (ed.) WADT 1997. LNCS, vol. 1376, pp. 223-237. Springer, Heidelberg (1998)

8. Gadducci, F., Heckel, R., Llabrés, M.: A bi-categorical axiomatisation of concurrent graph rewriting. In: Proc. CTCS 1999, Category Theory and Computer Science. Electronic Notes in Theoretical Computer Science, vol. 29, Elsevier Sciences, Amsterdam (1999)

9. Joyal, A., Street, R.H.: The geometry of tensor calculus I. Advances in Math. 88, 55-112 (1991)

10. Joyal, A., Street, R., Verity, D.: Traced monoidal categories. Mathematical Proceedings of the Cambridge Philosophical Society 119(3), 447-468 (1996)

11. Katis, P., Sabadini, N., Walters, R.F.C.: Bicategories of processes. Journal of Pure and Applied Algebra 115, 141-178 (1997)

12. Katis, P., Sabadini, N., Walters, R.F.C.: Span(Graph): A categorical algebra of transition systems. In: Johnson, M. (ed.) AMAST 1997. LNCS, vol. 1349, pp. 307321. Springer, Heidelberg (1997)

13. Katis, P., Sabadini, N., Walters, R.F.C.: On the algebra of systems with feedback and boundary. Rendiconti del Circolo Matematico di Palermo Serie II Suppl. 63, 123-156 (2000)

14. Katis, P., Sabadini, N., Walters, R.F.C.: A formalisation of the IWIM Model. In: Porto, A., Roman, G.-C. (eds.) COORDINATION 2000. LNCS, vol. 1906, pp. 267-283. Springer, Heidelberg (2000)

15. Katis, P., Sabadini, N., Walters, R.F.C.: Feedback, trace and fixed-point semantics. Theoret. Informatics Appl. 36, 181-194 (2002)

16. Kelly, G.M., Laplaza, M.L.: Coherence for compact closed categories. J. Pure Appl. Algebra 19, 193-213 (1980)

17. Kock, J.: Frobenius algebras and 2D topological Quantum Field Theories. Cambridge University Press, Cambridge (2004)

18. Menni, M., Sabadini, N., Walters, R.F.C.: A universal property of the monoidal 2-category of cospans of ordinals and surjections. Theory and Applications of Categories 18(19), 631-653 (2007)

19. Meseguer, J., Montanari, U.: Petri Nets Are Monoids. Information and Computation 88, 105-155 (1990)

20. Penrose, R.: Applications of negative dimensional tensors. In: Combinatorial Mathematics and its Applications, p. 221. Academic Press, London (1971)

21. Rosebrugh, R., Sabadini, N., Walters, R.F.C.: Minimization and minimal realization in Span(Graph). Mathematical Structures in Computer Science 14, 685-714 (2004)

22. Rosebrugh, R., Sabadini, N., Walters, R.F.C.: Generic commutative separable algebras and cospans of graphs. Theory and Applications of Categories 15(6), 264-277 (2005)

23. Rosebrugh, R., Sabadini, N., Walters, R.F.C.: Calculating colimits and limits compositionally. Category Theory 2007, Carvoeiro, Portugal, 18th (June 2007)

24. Rosebrugh, R., Sabadini, N., Walters, R.F.C.: Calculating colimits and limits compositionally (in preparation)

25. Walters, R.F.C.: Lecture to the Sydney Category Seminar (January 26, 1983)

26. Walters, R.F.C.: The tensor product of matrices, Lecture. In: International Conference on Category Theory, Louvain-la-Neuve (1987) 